POS PROCEEDINGS

\title{
Solar modulation of galactic cosmic ray electrons and positrons over the 23rd solar minimum with the PAMELA experiment.
}

Riccardo Munini; $^{* 1,2}$, Valeria di Felice ${ }^{3,4}$, M. Boezio ${ }^{2}$, O. Adriani ${ }^{5,6}$, G. C. Barbarino ${ }^{7,8}$, G. A. Bazilevskaya ${ }^{9}$,R. Bellotti ${ }^{10,11}$, E. A. Bogomolov ${ }^{12}$, M. Bongi $i^{4,6}$, V. Bonvicini ${ }^{2}$, S. Bottai ${ }^{6}$, A. Bruno ${ }^{10,11}$, F. Cafagna ${ }^{11}$, D. Campana ${ }^{8}$, P. Carlson ${ }^{13}$, M. Casolino ${ }^{3,15}$, G. Castellini ${ }^{16}$, C. De Donato ${ }^{3}$, C. De Santis ${ }^{14}$, N. De Simone ${ }^{3}$, V. Formato ${ }^{1,2}$, A. M. Galper ${ }^{17}$, A. V. Karelin ${ }^{17}$, S. V. Koldashov ${ }^{17}$, S. Koldobskiy ${ }^{17}$, S. Y. Krutkov ${ }^{12}$, A. N. Kvashnin ${ }^{9}$, A. Leonov ${ }^{17}$,V. Malakhov ${ }^{17}$, L. Marcelli ${ }^{14}$, M. Martucci ${ }^{14,18}$, A. G. Mayorov ${ }^{17}$, W. Menn ${ }^{19}$, M Merge ${ }^{3,14}$, V. V. Mikhailov ${ }^{17}$, E. Mocchiutti ${ }^{2}$, A. Monaco ${ }^{10,11}$, N. Mori ${ }^{6}$, G. Osteria ${ }^{8}$, F. Palma ${ }^{3,14}$, B. Panico ${ }^{8}$ P. Papini ${ }^{6}$, M. Pearce ${ }^{13}$, P. Picozza ${ }^{3,14}$, M. Ricci ${ }^{18}$, S. B. Ricciarini ${ }^{16}$, R. Sarkar ${ }^{2}$, V. Scotti ${ }^{7,8}$, M. Simon $^{19}$, R. Sparvoli ${ }^{3,14}$, P. Spillantini ${ }^{5,6}$, Y. I. Stozhkov ${ }^{9}$, A. Vacchi $^{2}$, E. Vannuccini ${ }^{6}$, G. Vasilyev ${ }^{12}$, S. A. Voronov ${ }^{17}$, Y. T. Yurkin ${ }^{17}$, G. Zampa ${ }^{2}$, N. Zampa ${ }^{2}$, M. S. Potgieter ${ }^{21}$, E.E. Vos ${ }^{21}$. 
${ }^{1}$ University of Trieste, Department of Physics, I-34147 Trieste, Italy

${ }^{2}$ INFN, Sezione di Trieste I-34149 Trieste, Italy

${ }^{3}$ INFN, Sezione di Rome "Tor Vergata”, I-00133 Rome, Italy

${ }^{4}$ Agenzia Spaziale Italiana (ASI) Science Data Center, I-00044 Frascati, Italy

5 University of Florence, Department of Physics, I-50019 Sesto Fiorentino, Florence, Italy

${ }^{6}$ INFN, Sezione di Florence, I-50019 Sesto Fiorentino, Florence, Italy

${ }^{7}$ University of Naples "Federico II", Department of Physics, I-80126 Naples, Italy

${ }^{8}$ INFN, Sezione di Naples, I-80126 Naples, Italy

${ }^{9}$ Lebedev Physical Institute, RU-119991, Moscow, Russia

${ }^{1} 0$ University of Bari, Department of Physics, I-70126 Bari, Italy

${ }^{11}$ INFN, Sezione di Bari, I-70126 Bari, Italy

12 Ioffe Physical Technical Institute, RU-194021 St. Petersburg, Russia

${ }^{13}$ KTH, Department of Physics, and the Oskar Klein Centre for Cosmoparticle Physics, AlbaNova University Centre, SE-10691 Stockholm, Sweden

${ }^{14}$ University of Rome "Tor Vergata”, Department of Physics, I-00133 Rome, Italy

${ }^{15}$ RIKEN, Advanced Science Institute, Wako-shi, Saitama, Japan

${ }^{16}$ IFAC, I-50019 Sesto Fiorentino, Florence, Italy

${ }^{17}$ National Research Nuclear University MEPhI, RU-115409 Moscow

${ }^{18}$ INFN, Laboratori Nazionali di Frascati, Via Enrico Fermi 40, I-00044 Frascati, Italy

${ }^{19}$ Universität Siegen, Department of Physics, D-57068 Siegen, Germany

${ }^{20}$ INFN, Sezione di Perugia, I-06123 Perugia, Italy

${ }^{21}$ North-West University, Centre for Space Research,2520 Potchefstroom, South Africa

E-mail: Riccardo.Munini@ts.infn.it

The satellite-borne PAMELA experiment has been continuously collecting data since 15th June 2006, when it was launched from the Baikonur cosmodrome to detect the charged component of cosmic rays over a wide energy range and with unprecedented statistics. The apparatus design is particularly suited for particle and antiparticle identification. The PAMELA experiment has measured the time variation of electron and positron spectrum at Earth in great detail, extending the measurement down to $70 \mathrm{MeV}$ and $200 \mathrm{MeV}$ respectively. The spectra have been evaluated with data collected from July 2006 to December 2009, i.e. during the $\mathrm{A}<0$ solar minimum of solar cycle 23, over six-months intervals. These spectra provide important information for the study of CR propagation inside the heliosphere and the investigation of the charge-dependent solar modulation.

The 34th International Cosmic Ray Conference,

30 July- 6 August, 2015

The Hague, The Netherlands

\footnotetext{
*Speaker.
} 


\section{Introduction}

The precise measurements of Cosmic Ray (CR) electrons and positron energy spectrum provide fundamental information regarding their origin and propagation. However the majority of the past measurements were obtained with experiments well inside the heliosphere. Therefore the effects of the propagation and modulation of galactic CR in the heliosphere cannot be neglected. As CRs traverse the turbulent magnetic field embedded into the solar wind particles are scattered by its irregularities and undergo convection and adiabatic deceleration in the expanding solar wind. Gradient and curvature drifts have also an effect, that is particularly important during periods of minimum solar activity. As a consequence the intensity of CR at Earth decreases with respect to the flux outside the heliosphere, the so called Local Interstellar Spectrum (LIS). Solar modulation has large effects on low energy CRs (less than a few GV) but have negligible effects on particles with rigidities grater than few tens of GV.

Furthermore, drift models predict a clear charge-sign dependence for the modulation of CRs [1], whose effects are expected to be particularly evident at energies below a few GeV. During so-called $\mathrm{A}<0$ polarity cycles like solar cycle 23 , when the heliospheric magnetic field is directed toward the Sun in the northern hemisphere, negatively-charged particles drift inward primarily through the polar regions of the heliosphere and outward along the heliospheric equator. Positively-charge particles drift in opposite direction. The situation reverses when the solar magnetic field changes its polarity at each solar maximum. This was shown by the $\left(\mathrm{e}^{-}+\mathrm{e}^{+}\right)$and proton measurements from few up to the $\mathrm{GeV}$ region by the KET instrument on board the Ulysses spacecraft [2] that explored the high latitude regions of the inner heliosphere from 1990 to 2009. Similar conclusion can be drawn by the electron $\left(\mathrm{e}^{-}+\mathrm{e}^{+}\right)$data collected by the balloon-born experiment LEE over several decades [3].

The most recent period of solar minimum activity and the consequent minimum modulation conditions for cosmic rays were unusual. It was expected that the new activity cycle would begin early in 2008. Instead solar minimum modulation conditions continued until the end of 2009 when the largest fluxes of galactic cosmic rays since the beginning of the space age were recorded [4]. Solar minimum activity is the ideal condition to study the modulation processes that affect the CR propagation inside the heliosphere.

PAMELA (Payload for Antimatter Matter Exploration and Light-nuclei Astrophysics) is a satellite-borne experiment designed to make long duration measurements of the cosmic radiation [5]. Results on the effects of the solar modulation on the energy spectra of galactic cosmic-ray protons in the period July 2006-December 2009 have already been published [6] by the PAMELA collaboration. Here we present new results on the long-term variation in the energy spectrum of galactic cosmic-ray electrons $\left(\mathrm{e}^{-}\right)$and positrons $\left(\mathrm{e}^{+}\right)$measured down to $70 \mathrm{MeV}$ and $200 \mathrm{MeV}$ respectively. This set of measurements allows to test the model which describes the CR propagation in the heliosphere and to quantify the contribution of the charge-sing dependence due to particle drift.

\section{The PAMELA instrument}

The PAMELA experiment was launched on June $15^{\text {th }} 2006$ from the Bajkonur cosmodrome 


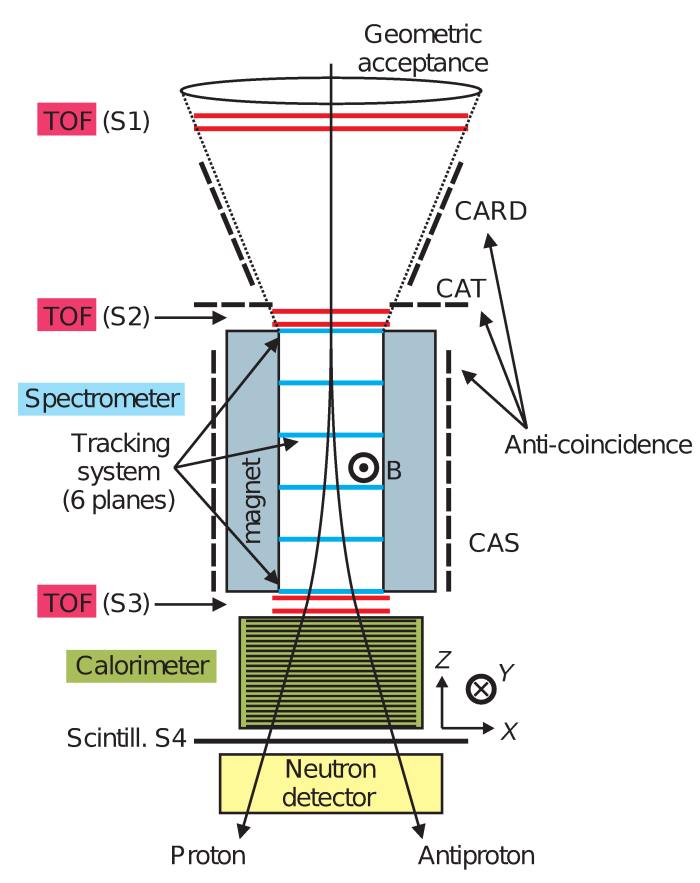

Figure 1: PAMELA and its sub-detectors.

on-board of the Resurs DK1 satellite and, since then, it has been almost continuously taking data. The instrument is following a high inclination $\left(70^{\circ}\right)$ orbit, ideal condition to observe the solar modulation of galactic cosmic rays down to very low energies $(<100 \mathrm{MeV})$.

The PAMELA spectrometer [5] was designed and built to study the antimatter component of cosmic rays from tens of $\mathrm{MeV}$ up to hundreds of $\mathrm{GeV}$ and with a significant increase in statistics with respect to previous experiments. To reach this goal the apparatus was optimized for the study of $\mathrm{Z}=1$ particles and to reach a high level of electron-proton discrimination.

The apparatus comprises the following subdetectors, arranged as shown schematically in Figure 1 (from top to bottom): a time-of-flight system (TOF S1, S2, S3); a magnetic spectrometer; an anticoincidence system (CARD, CAT, CAS); an electromagnetic imaging calorimeter; a shower tail catcher scintillator (S4) and a neutron detector.

The central components of PAMELA are a permanent magnet and a tracking system composed of six planes of double-sided silicon sensors, which form the magnetic spectrometer. The main task of the magnetic spectrometer is to measure the particle rigidity $\rho=\mathrm{pc} / \mathrm{Ze}$ (p and Ze being respectively the particle momentum and charge, and $\mathrm{c}$ the speed of light) and the ionization energy losses $(\mathrm{dE} / \mathrm{dx})$. The rigidity measurement is done through the reconstruction of the trajectory based on the impact points on the tracking planes and the resulting determination of the curvature due to the Lorentz force. The Time-of-Flight (ToF) system comprises three double layers of plastic scintillator paddles with the first two placed above and the third immediately below the magnetic spectrometer. The ToF system provides the measurements of the particle velocity combining the time of passage information with the track length derived from the magnetic spectrometer. By measuring the particle velocity, direction and curvature the spectrometer can distinguish between 


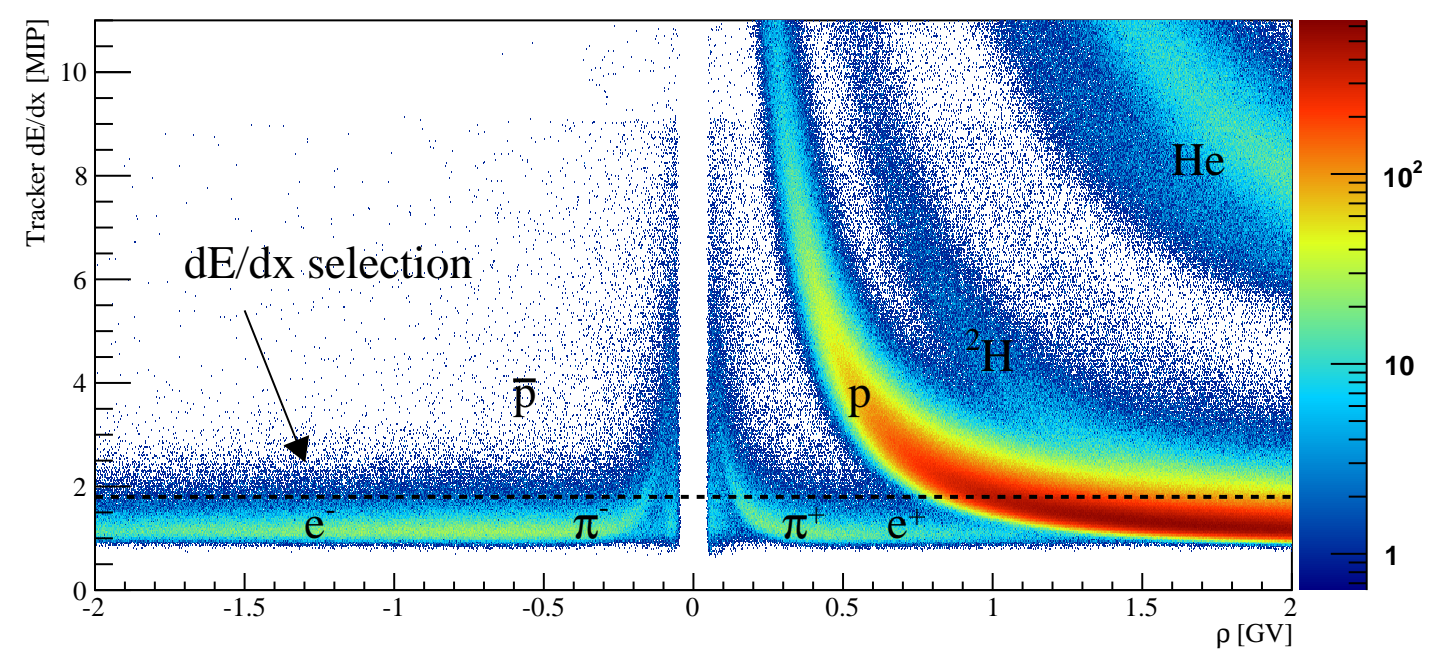

Figure 2: Rigidity distribution for the mean energy release on the tracker planes expressed in minimum ionizing particle units, MIP. On the positive side protons dominate. Below $300 \mathrm{MV}$ is clearly visible the secondary pion contamination. The dotted black line represents the charge one selection.

downgoing particles and upgoing splash ${ }^{1}$ albedo particles and separate negatively from positivelycharged particles.

The sampling imaging calorimeter (16.3 radiation lengths, 0.6 interaction lengths) is used for hadron-lepton separation, using topological and energetic informations about the shower development in the calorimeter. The shower tail catcher and the neutron detector beneath provide additional information for the discrimination. An anticoincidence system is used to reject spurious events in the off-line phase.

The total weight of PAMELA is $470 \mathrm{~kg}$ while the power consumption is $355 \mathrm{~W}$. A more detailed description of the instruments and the data handling can be found in [5].

\section{Data analysis}

A first selection on the goodness of the reconstructed track, expressed in terms of the $\chi^{2}$ of the fit, was made in order to obtain a sample of events with a reliable reconstructed rigidity. Only single track events were selected and a minimum of 3 impact points on the non bending view and 4 impact points on the bending view in the tracking system were required. Furthermore the track was required to be reconstructed inside a fiducial volume bounded $0.15 \mathrm{~cm}$ from the magnet cavity walls to increase the spectrometer performance. Splash albedo particles were rejected requiring $\beta>0$ 2 . Figure 2 illustrates the rigidity distribution for the mean ionization losses in the tracker planes for events selected as described above. For the electron and positron identification the following sources of contamination were studied:

\footnotetext{
${ }^{1}$ Upgoing secondary particles resulting from the interaction of high energy CRs with the atmosphere.

${ }^{2} \beta=v / c$ is defined as a the ratio between the particle velocity measured by the ToF system and the speed of light.
} 


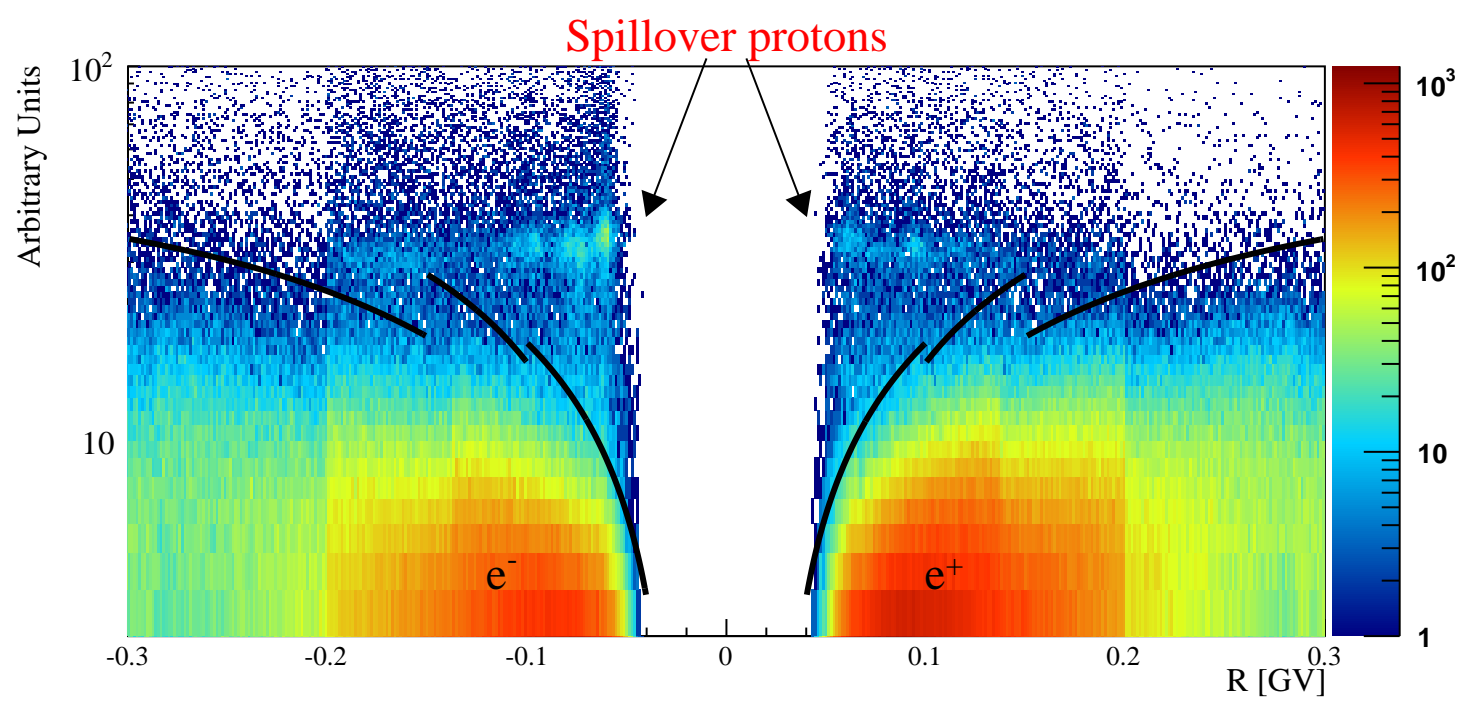

Figure 3: Calorimeter variable rigidity distribution. Below $300 \mathrm{MeV}$ on both the positively and negatively charged component a residual contamination of spillover protons can be seen. The black lines represent the selections used to remove the contamination.

- Protons: positrons are highly contaminated by protons. The positron to proton ratio decreases from $\sim 10^{-3}$ at $1 \mathrm{GeV}$ to $\sim 10^{-4}$ at $100 \mathrm{GeV}$.

- Anti-protons: the galactic anti-proton component represents for the electrons a contamination of a few percent over the entire rigidity range.

- Pions: this secondary component is locally produced by CRs, mostly protons, interacting with the PAMELA structure or pressure vessel. This component had already been studied for the anti-proton analysis [7]. Pion contamination reaches a maximum value around 200-300 MV amounting to a few percent of the electrons and positrons signal and decrease rapidly becoming negligible, according to simulation, above $1 \mathrm{GV}$. A slightly excess of positive pions is present due to charge conservation at the production reaction. This component is clearly visible below $300 \mathrm{MV}$ in Figure 2 both for positive and negative rigidities.

- Spillover protons: sometimes high energy $(>10 \mathrm{GV})$ protons are reconstructed as low energy $(<1 \mathrm{GV})$ positively or negatively charged particles. Because of the presence of spurious hits in the tracker planes a wrong curvature is assigned to the track. These events are significant at energies below $1 \mathrm{GeV}$ and amount to a few percent of the electrons signal (see Figure 3).

Requiring $\beta>0.9$, tracker $\mathrm{dE} / \mathrm{dx}<1.8 \mathrm{MIP}$ (see Figure 2) and ToF $\mathrm{dE} / \mathrm{dx}<3 \mathrm{MIP}$, protons and anti-protons were rejected up to $1 \mathrm{GeV}$ and pions up to $250 \mathrm{MeV}$. Moreover a large fraction of the pion events had hits in the top anticoincidence scintillators and it was removed requiring no activity on CARD and CAT. Being relativistic, spillover protons were unaffected by these selections.

The residual pion and proton contaminations were removed using the calorimeter informations. The calorimeter selections were developed using a Monte Carlo simulation of the PAMELA apparatus based on the GEANT4 code [8]. The simulation reproduces the entire PAMELA apparatus, including the pressure vessel, and was validated using particle beam data. The longitudinal 
and transverse segmentation of the calorimeter allow leptonic showers to be selected with high efficiency and small contamination above $300 \mathrm{MeV}$. The calorimeter electron selection was based on variables that emphasizes the differences between the leptonic and hadronic shower like the multiplication with increasing calorimeter depth and the collimation of the electromagnetic cascade along the track. Combining several of these variables anti-proton and pion contamination were reduced to a negligible amount up to tens of GV [9].

Since electromagnetic showers below $1 \mathrm{GeV}$ usually develop in the first half of the calorimeter instead of high energy protons that are non-interactive and traverse the entire volume, the spillover protons were rejected developing variables based on the energy deposit in the last planes of the calorimeter. Figure 3 shows one of these calorimeter variables. As shown in the picture a cut on this quantity rejected spillover protons without affecting significantly the electron and positron signal.

After all these selections, the residual contamination of pions, protons, anti-protons and spillover protons was estimated to be negligible over the entire energy range.

\section{Results}

The fluxes $\phi(\rho)$ are evaluated as follows:

$$
\phi(\rho)=\frac{1}{\varepsilon(\rho) \times G(\rho) \times T \times \Delta \rho} \times N
$$

where $N$ is the number of selected events, $\varepsilon(\rho)$ the overall selections efficiency, $G$ the geometrical factor, $T$ the live-time and $\Delta \rho$ the width of the rigidity bin.

The efficiencies were estimated using both simulated and flight data. Tracker efficiency was obtained entirely from simulation. The anticoincidence and ToF ionization losses efficiencies were evaluated with simulation using the real data both as cross-check and to evaluate possible normalization factors. The efficiencies of the $\beta$ and calorimeter selections were entirely evaluated using flight data.

The temporal dependence of the efficiencies was carefully studied. The most significant time variation was found in tracker efficiency that was estimated using the simulation and decrease with time due to a progressive deterioration of the read-out chips [6].

The live time was provided by an on-board clock that timed the periods during which the apparatus is waiting for a trigger. The geometrical factor was estimated with simulation to be constant at $19.9 \mathrm{~cm}^{2}$ sr down to $200 \mathrm{MeV}$, below this energy it decrease significantly due to the increase in the particles bending.

Electron rigidity spectra were obtained for different intervals of vertical geomagnetic cutoff rigidities, estimated in the Störmer approximation [10] using the satellite orbital information. Then spectra were unfolded using a Bayesian unfolding procedure [11] in order to take into account energy losses in the instrument and the tracker resolution on the rigidity reconstruction. In this way the energy spectra at the top of the payload were obtained. After the unfolding procedure the spectra were combined accounting for the proper live times and acceptance using only the fluxes at energies that exceeded 1.3 times the maximum vertical geomagnetic cutoff at each cutoff interval, thus excluding contamination from re-entrant albedo particles. 


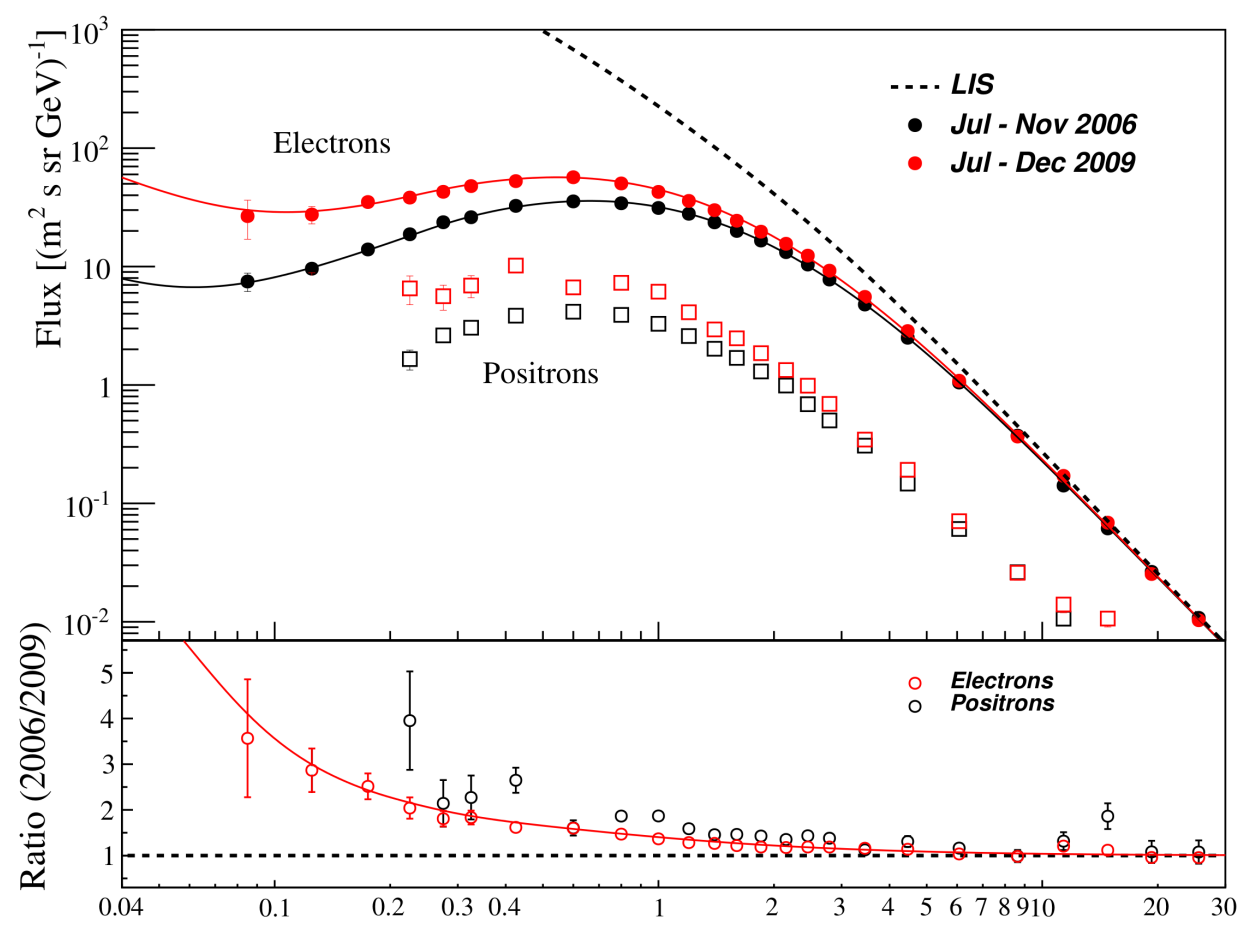

Figure 4: Top panel: July-November 2006 and July-December 2009 electron and positron energy spectra as measured by PAMELA. Bottom panel: ratio between the 2009 and 2006 fluxes. The black dotted line represents the electron LIS, the solid line is a fit with a 3D model for the electron propagation through the heliosphere [12]. The solar modulation is clearly visible and became negligible above $\sim 20 \mathrm{GeV}$. The error bars indicate only statistical uncertainties.

Figure 4 shows the electron and positron energy spectra for the periods July-November ${ }^{3} 2006$ and July-December 2009. The time dependence of the fluxes is clearly visible. This preliminary positrons analysis was limited down to $200 \mathrm{MeV}$. The bottom panel of Figure 4 shows the ratio between the 2009 and 2006 fluxes for both positrons and electrons. It follows from this ratio that the low-energy electron flux increased by a factor of about 2-3 from 2006 to 2009. Positrons at these energies, on the other hand, increased by a factor systematically higher over this period, indicating the effect of particle drifts.

\section{Conclusions}

We have presented new results on the electron and positron energy spectrum obtained by the PAMELA experiment during the past extra-ordinary solar minimum period that ended in late 2009 - beginning of 2010. The analysis for low energies positrons down to $70 \mathrm{MeV}$ is in progress and will be presented at the conference as well as the whole set of $\mathrm{e}^{-}$and $\mathrm{e}^{+}$fluxes.

\footnotetext{
${ }^{3}$ December 2006 was excluded from the analysis to prevent data contamination from particles created during the huge solar flare occurred on December 6.
} 


\section{References}

[1] M. S. Potgieter 2014 Adv. Space Res. 53 1415-1425

[2] B. Heber, M. S. Potgieter, 2006 Space Science Reviews. 127117

[3] P. Evenson, J. Clem, 2011 Proc. of the 32rd Int. Cosmic Ray Conf. (Beijing. China) 1152

[4] M. S. Potgieter et al. 2013 Proc. of the 33rd Int. Cosmic Ray Conf. (Rio de Janeiro. Brazil) arXiv:1308.1617 [astro-ph.SR]

[5] P. Picozza, et al. 2007 Astropart. Phys. 27296

[6] O. Adriani, et al. 2013 Astrophys. J. 76591

[7] O. Adriani, et al. 2009 Phys. Rev. Lett. 102051101

[8] S. Agostinelli et al. 2003 Nucl. Instrum. Meth. A 506250

[9] O. Adriani, et al. 2009 Nature 458 607-609

[10] M. A. Shea, D. F. Smart, and L. Gentile 1987 Physics of the Earth and Planetary Interiors 48200

[11] G. D’Agostini 1995 NIM A362 487-498

[12] M. S. Potgieter et al. 2015 submitted to Astrophys. J. 